Acta Botanica Mexicana (2002), 60: 1-5

\title{
DOS NUEVOS REGISTROS PARA LA FLORA DE MUSGOS DE COSTA RICA: STEEREOBRYON SUBULIROSTRUM Y POGONATUM PENSILVANICUM \\ (POLYTRICHACEAE)
}

\author{
EFRAÍN DE LUNA \\ Departamento de Sistemática Vegetal \\ Instituto de Ecología, A.C. \\ 91070 Xalapa, Veracruz, México \\ María Silvina Ussher Miozzo \\ Universidad de los Andes \\ Centro Jardín Botánico \\ Mérida, Venezuela \\ Y \\ Raquel Cobos Acosta \\ Universidad Nacional de Colombia \\ Bogotá, D.C. Colombia
}

\begin{abstract}
RESUMEN
Como resultado de exploraciones durante el Primer Curso de "Briología Tropical" de la Red Latinoamericana de Botánica, se recolectaron dos especies de Polytrichaceae que son nuevas para la flora de musgos de Costa Rica y América Central: Steereobryon subulirostrum (Schimp. ex Besch.) G. L. Sm. y Pogonatum pensilvanicum (Hedw.) P. Beauv. El primer taxon además representa un género no conocido previamente de Centroamérica. En tal virtud, se proveen claves para la identificación de los géneros de Polytrichaceae y de las especies de Pogonatum de Costa Rica.
\end{abstract}

Palabras clave: briofitas, Costa Rica, florística, musgos, Polytrichaceae.

\section{ABSTRACT}

As a result of field trips during the first "Tropical Bryology" course of the Red Latinoamericana de Botánica, we collected two species of Polytrichaceae that are new to the moss flora of Costa Rica and Central America: Steereobryon subulirostrum (Schimp. ex Besch.) G. L. Sm. and Pogonatum pensilvanicum (Hedw.) P. Beauv. The former taxon represents also a genus previously not known from Central America. In this connection we provide two identification keys, one for the genera of Polytrichaceae, and another for species of Pogonatum in Costa Rica.

Key words: bryophytes, Costa Rica, floristics, mosses, Polytrichaceae. 


\section{INTRODUCCIÓN}

Entre los países centroamericanos, posiblemente la flora de musgos de Costa Rica es la mejor conocida y la más diversa. Inicialmente Bowers (1974) reconoció 542 taxa. Los registros adicionales de Crosby (1974) y Morales (1982), así como los rearreglos taxonómicos y nomenclaturales modernos, permiten reajustar la lista a más de 620 especies de musgos (Dauphin y Morales, com pers.; Delgadillo et al. 1995).

Gracias al apoyo de la Red Latinoamericana de Botánica (RLB), un grupo de briólogos latinoamericanos participamos en una excursión a la estación biológica "Alberto M. Brenes", en la región central de Costa Rica. Este viaje de campo se realizó como parte del primer curso de "Briología Tropical" de la RLB, efectuado en la Universidad de Costa Rica, San José. Entre las colecciones destacan las que aquí se comunican por representar dos adiciones a la flora conocida de los musgos del mencionado país.

\section{RESULTADOS Y DISCUSIÓN}

Steereobryon subulirostrum (Schimp. ex Besch.) G. L. Sm., Mem. New York Bot. Gard. 21: 56. 1971.

Espécimen y localidad: De Luna 2576 (USJ, XAL), Alajuela, Estación Biológica "A. M. Brenes", UCR. NE de San Ramón. Bosque premontano nuboso con Lauraceae, Heliocarpus, Wercklea y estrato arbustivo muy desarrollado. A la orilla del camino. Sobre suelo entre rocas bajas, sitio expuesto y húmedo. Altitud ca. $800 \mathrm{~m}$. Febrero 16, 2001.

Este es el primer registro del género para Costa Rica y para Centroamérica. Todavía no se ha resuelto si se debe reconocer dos especies de Steereobryon o una sola. Por un lado Crosby et al. (1992) y Churchill et al. (2000) listan a: S. elamellosum (Herzog) Menzel y $S$. subulirostrum, ambas distribuidas en el Neotrópico. Sin embargo, Smith (1994) e Hyvönen et al. (1998) aluden al género como monotípico, reconociendo solamente a $S$. subulirostrum.

Si Steereobryon (Polytrichaceae) tiene dos especies, entonces S. elamellosum (Herzog) Menzel no se ha recolectado en ningún otro país (Churchill et al., 2000) desde que se describió originalmente de Bolivia. Delgadillo et al. (1995) la citan como Atrichum elamellosum (Herzog) Frye \& Duck., aunque el taxon ya se había transferido a Steereobryon (cf. Crosby et al. 1992; Smith, 1994). Se puede hallar una descripción detallada e ilustraciones de $S$. elamellosum en Frye y Duckering (1947).

La segunda especie del género es $S$. subulirostrum. Se ha recolectado en Colombia, Ecuador, Venezuela (Churchil y Linares, 1995), Perú (Churchill et al., 2000), México (Smith, 1994), República Dominicana (Buck y Steere, 1983) y Puerto Rico (Sastré De Jesús y Buck, 1993). Además, Smith (1971) la cita de Jamaica, aunque Delgadillo et al. (1995) omiten este dato, por lo que su presencia en Jamaica debe ser confirmada. El registro de $S$. subulirostrum de Centroamérica se encontraba en una situación confusa similar. Smith (1971) no cita ningún país de esta parte del continente en la distribución del taxon. Después, Smith (1994) y Delgadillo et al. (1995) lo aceptaron como ya registrado de América Central, pero no especificaron el país. Ahora se ha recolectado en 
Costa Rica y se confirma así su presencia en el área. Se pueden hallar descripciones detalladas e ilustraciones de S. subulirostrum en Frye (1948) y Smith $(1971,1994)$.

Con objeto de facilitar la identificación de los géneros de Polytrichaceae conocidos de Costa Rica, presentamos una clave, adaptada de la de Griffin y Morales (1983) y la propuesta por Churchill y Linares (1995) con algunas modificaciones. Esta clave incluye Steereobryon y los seis géneros previamente conocidos de Costa Rica (Delgadillo et al. 1995).

Clave para los géneros de Polytrichaceae de Costa Rica

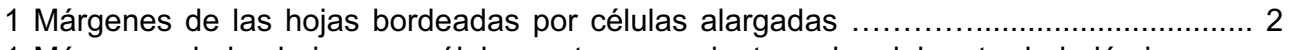

1 Márgenes de las hojas con células cortas, semejantes a las del resto de la lámina ......

2 Márgenes de las hojas con dientes simples; células del margen levemente pluripapilosas.... Steereobryon

2 Márgenes con dientes dobles; células del margen lisas, sin papilas .......... Atrichum

3 Márgenes de la hoja doblados longitudinalmente, cubriendo la superficie adaxial de la lámina; células terminales de las lamelas hendidas o piriformes Polytrichum

3 Márgenes planos o incurvados, superficie adaxial libre, células terminales de las lamelas de diversas formas, redondeadas, truncadas o piriformes ........................... 4

4 Lamelas restringidas a la superficie dorsal de la costa ........................ Oligotrichum

4 Lamelas sobre la costa y la superficie adaxial de la lámina .................................. 5

5 Hojas claramente diferenciadas en vaina y limbo, vaina obovada a oblongo-obovada; cápsula angular a prismática, peristoma con 64 dientes

5 Hojas levemente o no diferenciadas en vaina y limbo, vaina ovada; cápsula rolliza a cilíndrica, peristoma con 32 dientes .............................................................. Pogonatum

6 Células terminales de las lamelas papilosas, vaina de la hoja semitranslúcida o verdosa; cápsulas con 4 ángulos; caliptra densamente pilosa ........ Polytrichastrum

6 Células terminales de las lamelas lisas, vaina de la hoja teñida de anaranjado; cápsulas con 2 ángulos; caliptra glabra o escasamente pilosa ....... Polytrichadelphus

Pogonatum pensilvanicum (Hedw.) P. Beauv., Mém. Soc. Linn. Paris 1: 461. 1822.

Espécimen y localidad: De Luna 2575, (USJ, XAL), Alajuela, Estación Biológica "A. M. Brenes", UCR. NE de San Ramón. Bosque premontano nuboso con Lauraceae, Heliocarpus, Wercklea y estrato arbustivo muy desarrollado. A la orilla del camino. Sobre suelo, talud expuesto y húmedo. Altitud ca. 800 m. Febrero 16, 2001.

Este es el primer registro de $P$. pensilvanicum para Costa Rica y para Centroamérica. La especie se ha citado de Canadá, Estados Unidos, México, Perú, Bolivia, Brasil, Paraguay, Cuba (Hyvönen, 1989; Delgadillo et al., 1995), Venezuela (Ramírez y Crusco de Dall'Aglio, 1978) y Colombia (Churchill y Linares, 1995; Churchill et al., 2000). Se pueden encontrar descripciones e ilustraciones en publicaciones recientes como las de Crum y Anderson (1981, p. 1262 fig. 627 A-I), Hyvönen (1989), Smith (1994) y Churchill y Linares (1995). El habitat en Costa Rica, un talud a la orilla 
del camino, corrobora la percepción de que $P$. pensilvanicum es común en ambientes ruderales y suelos expuestos.

Este taxon se agrega a otros del género ya conocidos en el área centroamericana. Pogonatum (Polytrichaceae) está formado por unas 52 especies en el mundo (Hyvönen, 1989), de las cuales, unas 10 son propias del Neotrópico. Para Costa Rica solamente se sabía de la existencia de cuatro: $P$. campylocarpum (Müll. Hal.) Mitt., $P$. comosum (Müll. Hal.) Mitt., $P$. procerum (Lindb.) Schimp. y P. tortile (Sw.) Brid. De Centroamérica se han registrado además otras dos: $P$. perichaetiale subsp. oligodus (Müll. Hal.) Hyvönen y $P$. volvatum (Müll. Hal.) Par. (Hyvönen 1989). La clave que se presenta a continuación para las cinco especies de Pogonatum conocidas de Costa Rica fue adaptada de Hyvönen (1989):

Clave para las especies de Pogonatum (Polytrichaceae) de Costa Rica

1 Hojas pequeñas o reducidas, lamelas vestigiales; protonema filamentoso persistente ...... P. pensilvanicum

1 Hojas anchas y bien desarrolladas, lamelas usualmente bien desarrolladas; protonema efímero

2 Células apicales de las lamelas con paredes delgadas, isodiamétricas ................ 3

2 Células apicales de las lamelas con paredes engrosadas, claramente más altas que anchas

3 Gametofitos robustos, células apicales de las lamelas geminadas (dobles)

P. procerum

3 Gametofitos de tamaño mediano, células apicales de las lamelas mayormente sencillas

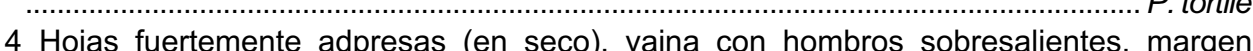
de la base entero; lámina con constricción basal acentuada; células apicales de las lamelas con la pared externa engrosada P. comosum

4 Hojas libremente curvadas (en seco), vaina sin hombros, márgenes de la base aserrados; lámina con constricción basal débil o ausente; células apicales de las lamelas con la pared externa ligeramente engrosada

P. campylocarpum

Las dos especies de Polytrichaceae tratadas en este trabajo son abundantes localmente en la estación biológica "A. M. Brenes". Dado el ambiente y el tipo de vegetación donde se recolectaron, es de esperarse que ambas tengan una distribución más amplia, especialmente en las regiones central y occidental de Costa Rica. Los presentes registros llenan parcialmente el vacío de la distribución conocida de ambas especies entre Sudamérica y Norteamérica.

\section{AGRADECIMIENTOS}

Las colecciones se efectuaron con financiamiento de la Red Latinoamericana de Botánica para el curso "Briología Tropical", realizado en San José, del 11 al 22 de febrero, 2001. Apreciamos mucho el apoyo recibido de M. I. Morales para visitar la estación 
biológica "A. M. Brenes" y su ayuda en el envío de especímenes. Agradecemos los comentarios de Jaakko Hyvönen y Maria Isabel Morales y también las correcciones de dos revisores anónimos que mejoraron el manuscrito.

\section{LITERATURA CITADA}

Bowers, F. D. 1974. The mosses reported from Costa Rica. Bryologist 77: 150-171.

Buck, W. R. y W. C. Steere. 1983. Un listado preliminar de los musgos de La Española. Moscosoa 2: 28-53.

Churchill, S. P. y E. L. Linares. 1995. Prodromus bryologiae Novo-Granatensis. Introducción a la flora de musgos de Colombia. Biblioteca José Jerónimo Triana 12: 1-924.

Churchill, S., D. Griffin III y J. Muñoz. 2000. A checklist of the mosses of the tropical Andean countries. Ruizia 17: 1-202.

Crosby, M. R. 1974. Noteworthy Costa Rican mosses. Brenesia 4: 9-15.

Crosby, M. R., R. E. Magill y C. H. Bauer. 1992. Index of mosses 1963-1989. Monogr. Syst. Bot. Missouri Bot. Gard. 42: 1-646.

Crum, H. A. y L. E. Anderson. 1981. Mosses of eastern North America. Columbia University Press. Nueva York. 1328 pp.

Delgadillo, C., B. Bello y A. Cárdenas. 1995. LATMOSS. A catalogue of neotropical mosses. Monogr. Syst. Bot. Missouri Bot. Gard. 56: 1-191.

Frye, T. C. y M. W. Duckering. 1947. Atrichum elamellosum. Bryologist 50: 80-82.

Frye, T. C. 1948. Atrichum subulirostrum. Bryologist 51: 186-188.

Griffin, D. III y M. I. Morales. 1983. Keys of the genera of mosses from Costa Rica. Brenesia 21: 299-323.

Hyvönen, J. 1989. A synopsis of genus Pogonatum (Polytrichaceae, Musci). Acta Bot. Fennica 138: 1-87.

Hyvönen, J., T. A. Hedderson, G. L. Smith-Merril, J. G. Gibbings y S. Koskinen. 1998. On phylogeny of the Polytrichales. Bryologist 101: 489-504.

Morales, M. I. 1982. Musgos de Costa Rica: nuevos registros. Brenesia 19/20: 231-239.

Ramírez Reyes C. y R. Crusco de Dall'Aglio. 1978. Adiciones a la brioflora de Venezuela. Agronomía Tropical 10: 429-430.

Sastré De Jesús, I. y W. R. Buck. 1993. Annotated checklist of the mosses of Puerto Rico. Caribbean J. Sci. 29: 226-234.

Smith, G. L. 1971. A conspectus of the genera of Polytrichaceae. Mem. New York Bot. Gard. 21(3): 1-83.

Smith, G. L. 1994. Subclass Polytrichidae. In: Sharp, A. J., H. Crum y P. M. Eckel (eds.). The moss flora of Mexico. Mem. New York Bot. Gard. 69: 1068-1092. 